\title{
Influence of material defects on current-driven vortex domain wall mobility
}

\author{
Jonathan Leliaert, ${ }^{1,2, *}$ Ben Van de Wiele, ${ }^{1}$ Arne Vansteenkiste, ${ }^{2}$ Lasse Laurson, ${ }^{3}$ Gianfranco Durin,, 4 \\ Luc Dupré, ${ }^{1}$ and Bartel Van Waeyenberge ${ }^{2}$ \\ ${ }^{1}$ Department of Electrical Energy, Systems and Automation, Ghent University, Ghent B-9000, Belgium \\ ${ }^{2}$ Department of Solid State Science, Ghent University, Krijgslaan 281/S1, 9000 Ghent, Belgium \\ ${ }^{3}$ COMP Centre of Excellence and Helsinki Institute of Physics, Department of Applied Physics, \\ Aalto University School of Science, P.O. Box 11100, FI-00076 AALTO, Finland \\ ${ }^{4}$ Istituto Nazionale di Ricerca Metrologica, Strada delle Cacce 91, 10135 Torino, Italy \\ ${ }^{5}$ ISI Foundation, Via Alassio 11/c, 10126, Torino, Italy
}

(Received 24 October 2013; revised manuscript received 2 February 2014; published 24 February 2014)

\begin{abstract}
Many future concepts for spintronic devices are based on the current-driven motion of magnetic domain walls through nanowires. Consequently a thorough understanding of the domain wall mobility is required. However, the magnitude of the nonadiabatic component of the spin-transfer torque driving the domain wall is still debated today as various experimental methods give rise to a large range of values for the degree of nonadiabaticity. Strikingly, experiments based on vortex domain wall motion in magnetic nanowires consistently result in lower values compared to other methods. Based on the micromagnetic simulations presented in this contribution we can attribute this discrepancy to the influence of distributed disorder which vastly affects the vortex domain wall mobility, but is most often not taken into account in the models adopted to extract the degree of nonadiabaticity.
\end{abstract}

DOI: 10.1103/PhysRevB.89.064419

PACS number(s): 75.30.Hx, 75.78.Cd, 75.78.Fg

\section{INTRODUCTION}

In many future spintronic devices information is stored and processed by means of magnetic domain walls moving through magnetic nanowires [1-3]. Here, electrical currents are able to drive the magnetic domain walls by means of the spin-transfer torque interaction. To correctly describe this interaction, next to an adiabatic term also a nonadiabatic term [4] should be added to the Landau-Lifshitz equation,

$$
\dot{\mathbf{m}}=\gamma \mathbf{H}_{\mathrm{eff}} \times \mathbf{m}+\alpha \mathbf{m} \times \dot{\mathbf{m}}-[\mathbf{u} \cdot \nabla] \mathbf{m}+\beta \mathbf{m} \times[\mathbf{u} \cdot \nabla] \mathbf{m} .
$$

Here $\mathbf{m}$ is the magnetization, $\gamma$ the gyromagnetic ratio, $\alpha$ the Gilbert damping constant, $\mathbf{u}$ a velocitylike term proportional to the current density $J$, and $\beta$ the degree of nonadiabaticity. Since the introduction of this nonadiabatic term, there has been a lot of debate on the magnitude of $\beta$, with theoretically predicted values ranging from $\beta \approx \alpha$ [4-6] over $\beta=2 \alpha$ [7] to $\beta=4 \alpha$ [8]. Additionally, experiments have been until now unable to converge to one value.

Several experimental techniques have been used to quantify $\beta$. One way is to measure the depinning field to pull a vortex out of a pinning potential in the presence of a spin-polarized current [9-11]. A similar technique consists of looking at the thermal hopping between pinning sites in the presence of a spin-polarized current [12] where different values for $\beta$ for the same material are estimated depending on the considered magnetic structure: a vortex domain wall or a transverse domain wall. Another approach is to determine local vortex core displacements due to spin-polarized currents in the confining potential of, e.g., a pinning site [13], a disk [14], or a square geometry [15]. A third set of experiments, only able to extract $\beta / \alpha$, is based on measuring the distance a domain wall is able to cover due to a current pulse with known amplitude and duration. Here, resulting time and space

\footnotetext{
*jonathan.leliaert@ugent.be
}

averaged velocities are fitted to theoretical and/or simulated values [16-19]. Apart from these methods to directly quantify $\beta$ or $\beta / \alpha$, electrical and magnetic imaging techniques show domain wall transformations when an electric current is applied, indicating $\beta \neq \alpha$ [20-22]. Table I gives an overview of experiments performed to measure $\beta$.

Even within the broad range of possible values reported, a clear discrepancy between measurements based on domain wall motion and other methods is present. Here we show that these seemingly discrepant values for $\beta$ can be ascribed to the influence of distributed disorder on the time and space averaged motion of the magnetic domain wall, giving rise to an apparent degree of nonadiabaticity $\beta \approx \alpha$ irrespective of the actual value of $\beta$. This is supported by simulations of the motion of vortex domain walls in nanowires including the effect of realistic distributed disorder.

Simulations investigating the effect of sample imperfections on the domain wall mobility have mainly concentrated on nanowire edge roughness [23,24]. It is found that this suppresses the Walker breakdown (defined by the maximal linear motion of the transverse domain wall), allowing the domain wall to move faster for higher applied fields or currents compared to the corresponding nanowire with perfect geometry. These studies however neglect the influence of disorder distributed within the wire. Nevertheless, real Permalloy nanowire samples contain defects in their microstructure, e.g., surface roughness and/or grain boundaries, which can act as pinning centers for the domain walls. From experiments [25-29] it is known that these are randomly distributed throughout the wire with a density $\sigma$ ranging from 690 to $2000 \mu \mathrm{m}^{-2}$, and give rise to a pinning potential for a vortex that is approximately $2 \mathrm{eV}$ deep and has an interaction range roughly equal to the size of the vortex core. In this contribution we numerically investigate the influence of such distributed disorder on the domain wall mobility. 
TABLE I. Overview of experimentally obtained values for $\beta$ in Permalloy.

\begin{tabular}{lccc}
\hline \hline Method & $\beta$ & $\beta / \alpha$ & Ref. \\
\hline Current-assisted domain & $0.040 \pm 0.005$ & $2^{\mathrm{a}}$ & {$[9]$} \\
wall depinning from a & $0.040 \pm 0.005$ & $2-4^{\mathrm{a}}$ & {$[10]$} \\
pinning site & $0.040 \pm 0.0025$ & $\approx 5.3$ & {$[11]$} \\
Thermal depinning & & & \\
vortex domain wall & $0.073 \pm 0.026$ & $\approx 9$ & {$[12]$} \\
transverse domain wall & $0.01 \pm 0.004$ & $\approx 1$ & {$[12]$} \\
Local vortex core & 0.04 & $8^{\mathrm{a}}$ & {$[13]$} \\
movements & $0.15 \pm 0.07$ & $>10$ & {$[14]$} \\
& $0.15 \pm 0.02$ & $>7$ & {$[15]$} \\
Vortex domain wall & $0.01^{\mathrm{a}}$ & $0.96 \pm 0.02$ & {$[16]$} \\
motion in & $0.008^{\mathrm{a}}$ & 1 & {$[17]$} \\
nanowires & $0.007^{\mathrm{a}}$ & 0.7 & {$[18]$} \\
& Not mentioned & 1 & {$[19]$} \\
\hline \hline
\end{tabular}

${ }^{a}$ Based on estimated values of $\alpha$.

\section{MICROMAGNETIC METHODS}

The micromagnetic simulations are performed using the software package [30] MUMAX ${ }^{3}$. The domain wall motion is simulated in nanowires of width $400 \mathrm{~nm}$ and thickness $10 \mathrm{~nm}$ for $500 \mathrm{~ns}$, corresponding to a maximum wire length of $500 \mu \mathrm{m}$. A discretization of $3.125 \times 3.125 \times 10 \mathrm{~nm}^{3}$ is used. The window in which the magnetization is calculated is $1200 \mathrm{~nm}$ wide and moves with the domain wall. Additionally, typical material parameters for Permalloy are used: saturation magnetization $M_{s}=860 \times 10^{3} \mathrm{~A} / \mathrm{m}$, Gilbert damping $\alpha=$ 0.02 , and exchange stiffness $A=13 \times 10^{-12} \mathrm{~J} / \mathrm{m}$. To see the influence of $\beta$ on the domain wall dynamics, values $\beta=0$, $\beta=\alpha$, and $\beta=2 \alpha$ are considered. Here, the magnetic charges at the ends of the nanowire are compensated to simulate an infinitely long wire. For different current densities, the average domain wall velocity is computed based on the distance traveled by the wall during the simulation time. To include distributed defects in our simulations we introduced small regions $\left(9.375 \times 9.375 \mathrm{~nm}^{2}\right.$ in size $)$ with a reduced exchange length

$$
l_{\mathrm{ex}}=\sqrt{\frac{2 A}{\mu_{0} M_{s}^{2}}}
$$

at their boundaries. By reducing the exchange constant $A$ to $30 \%$ of its normal value across the region boundaries, a corresponding reduction in $l_{\mathrm{ex}}$ of roughly $50 \%$ was obtained. This method is a realistic way to include distributed defects that are reminiscent of material grains [31].

In Fig. 1 the pinning potential for such a single region is shown, illustrating the correspondence with experimental values. Random distributions of these regions with densities ranging from $\sigma=500$ to $1500 \mu \mathrm{m}^{-2}$ were included. This differs from earlier approaches to simulate distributed disorder [32,33]. In Ref. [32] disorder was introduced in the material by introducing void cells. Reference [33], on the other hand, implemented disorder by introducing slight variations in the saturation magnetization.

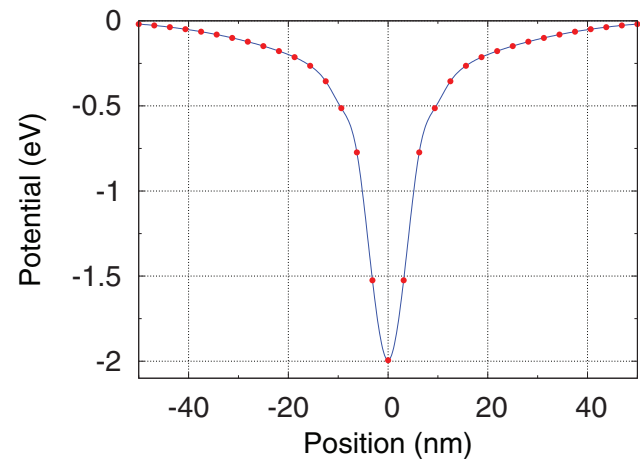

FIG. 1. (Color online) Potential well of a defect of size $3 \times 3$ discretization cells interacting with a vortex core, with the exchange length reduced by roughly $50 \%$ at the boundaries. The depth of the potential is approximately $2 \mathrm{eV}$ and the interaction range is comparable to the size of the vortex core diameter [31].

\section{RESULTS AND DISCUSSION}

Figure 2 shows averaged domain wall velocities $v$ versus applied current density $J$. Comparing the domain wall velocity in the disordered nanowires to the perfect nanowire case (solid lines) we observe (i) a depinning threshold at much smaller currents than the intrinsic depinning threshold, for the adiabatic as well as for the nonadiabatic case, and (ii) an absence of the Walker breakdown for the nonadiabatic case (here $\beta=2 \alpha$ ). To find the origin of this very different behavior we compare the domain wall motion in a perfect wire and in a nanowire with distributed disorder; see Figs. 3 and 4.

\section{A. Perfect nanowires}

In a perfect wire, the vortex core moves towards the nanowire edge in the direction defined by the vortex core polarization (for $\beta \neq \alpha$ ). Below the intrinsic depinning threshold (for $\beta=0$ ) or the Walker breakdown (for $\beta>0$ )

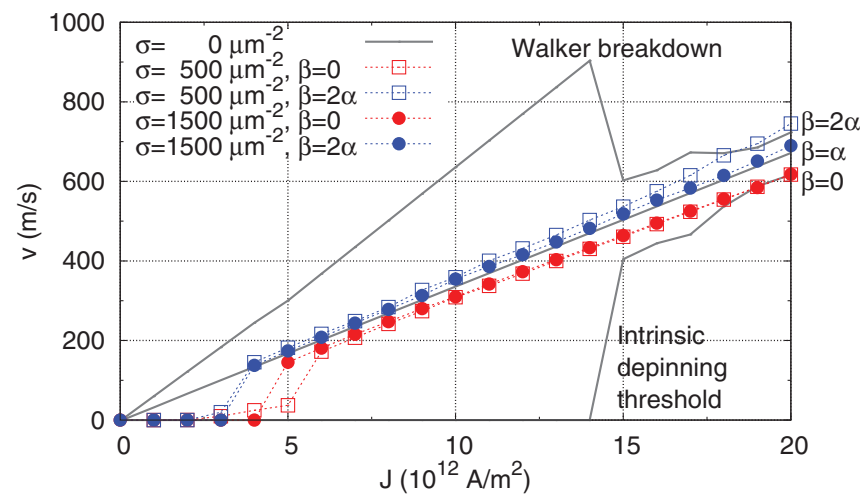

FIG. 2. (Color online) Velocity vs applied current density. Solid lines: velocity vs applied current density in a perfect nanowire $(\beta=0$, $\alpha$, and $2 \alpha$ ). Colored symbols: velocity vs applied current density in nanowires with disorder for $\beta=0$ (red) and $\beta=2 \alpha$ (blue). Irrespective of the used value of $\beta$, the velocity curves tend to converge to a case corresponding to $\beta=\alpha$ in perfect wires. For small applied current densities, extrinsic pinning of the vortex core on a defect takes place. 

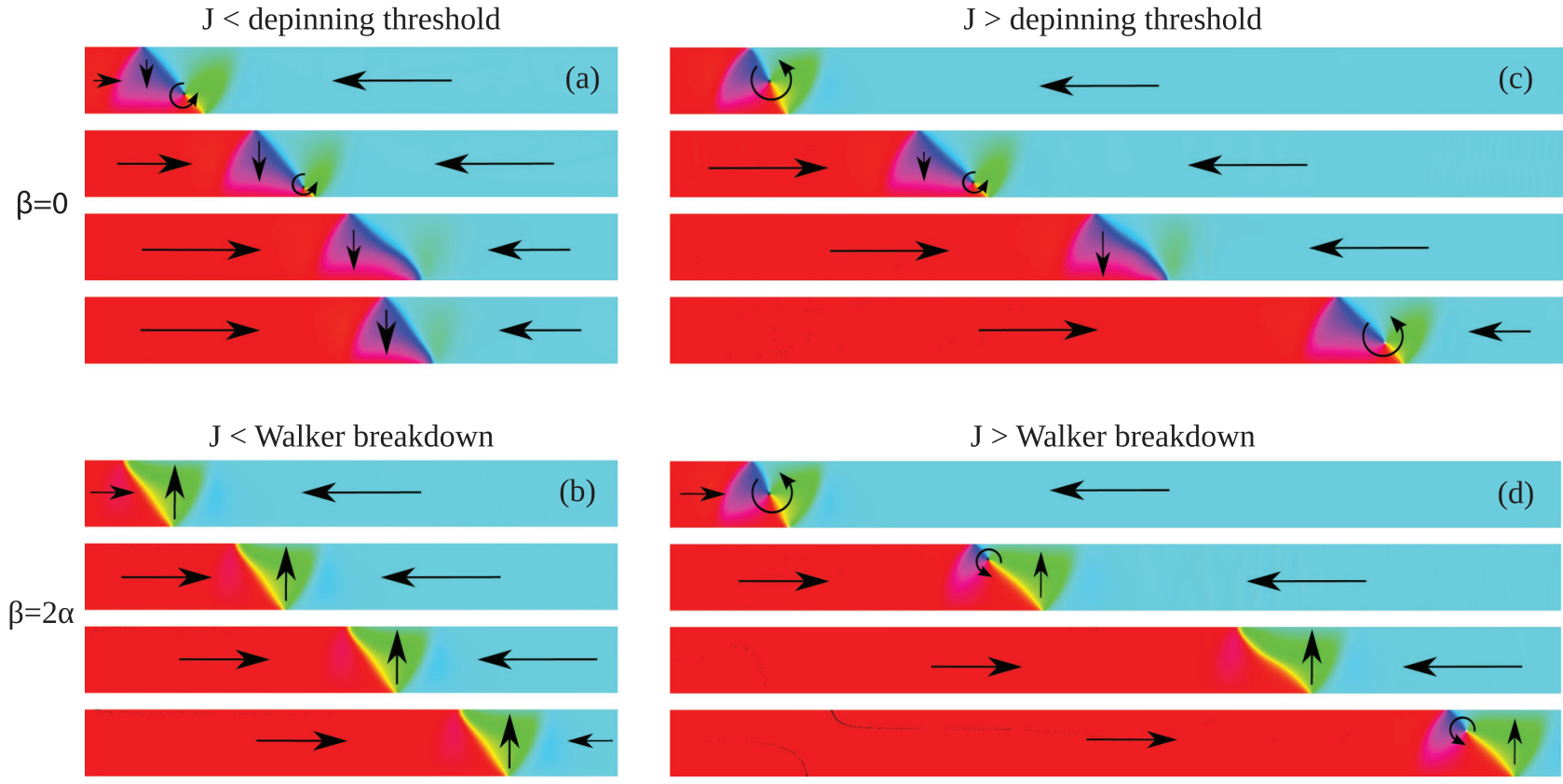

FIG. 3. (Color online) Snapshots of vortex domain wall motion in a perfect Py nanowire, $400 \mathrm{~nm}$ wide and $10 \mathrm{~nm}$ thick. While propagating, the vortex core moves towards the nanowire edge and the domain wall reshapes into a transverse domain wall. For small currents this wall gets intrinsically pinned, $\beta=0$ (a), or moves linearly with a speed proportional to applied current density, $\beta=2 \alpha$ (b). For large applied currents, the wall transforms again into a vortex domain wall, $\beta=0$ (c) and $\beta=2 \alpha(\mathrm{d})$. Now the core has an opposite polarization and moves consequently towards the opposite nanowire edge giving rise to successive domain wall transformations.

the vortex domain wall reshapes into a transverse domain wall which gets intrinsically pinned or moves linearly with applied current density, respectively (see Movies M1 and M2 in the Supplemental Material [34]). Above the Walker breakdown/depinning threshold the vortex core switches polarization and moves to the opposite nanowire edge (see Movies M3 and M4 in the Supplemental Material [34]). For $\beta=\alpha$ the vortex core moves perfectly along the nanowire center.
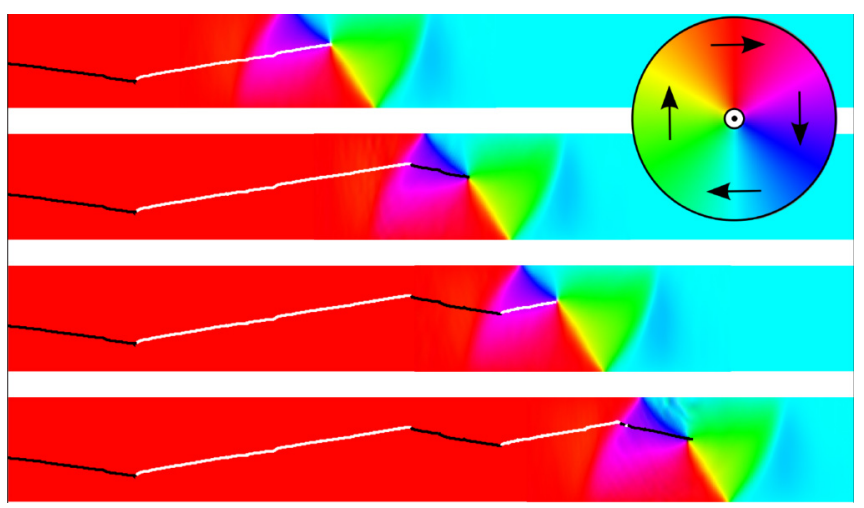

FIG. 4. (Color online) Snapshots of vortex domain wall motion in a disordered Py nanowire, $400 \mathrm{~nm}$ wide and $10 \mathrm{~nm}$ thick. The successive magnetization snapshots, at 3 ns time intervals, show a vortex wall driven adiabatically $(\beta=0)$ with current density $J=$ $10 \times 10^{12} \mathrm{~A} / \mathrm{m}^{2}$ in a nanowire with disorder density $\sigma=500 \mu \mathrm{m}^{-2}$. The vortex core trajectory is represented by the white/black lines, indicating a positive/negative vortex core polarization.

\section{B. Disordered nanowires}

In a disordered nanowire (see Fig. 4 and Movies M5 and M6 in the Supplemental Material [34]), the vortex core can switch polarization at a defect, implying a change in lateral propagation direction and thus hindering the formation of the transverse domain wall. This polarization switching mechanism, which was not found [35] in Ref. [33], explains the absence of the Walker breakdown and the much smaller depinning threshold. The pinning mechanism itself is also affected by the disorder: instead of the intrinsic pinning mechanism induced by the internal balancing of the effective field and spin-transfer torques inside the transverse domain wall found in a perfect nanowire, disorder gives rise to an extrinsic pinning mechanism in which the vortex core gets pinned at a defect. In the experimentally accessible current ranges, we observe an average motion of the vortex core in the central region of the wire without the formation of transverse domain walls due to successive core switches at defects. This resembles the motion of a vortex domain wall in a perfect wire for the case $\beta=\alpha$, which explains the values of $\beta / \alpha$ derived from domain wall motion (Table I).

Following the work of Ref. [32] we have also investigated the effect of voids on the domain wall mobility and have found qualitatively the same mobility curves. This can be explained by the fact that the pinning potentials caused by this type of disorder are much deeper [31], and therefore also allow vortex core polarization switching, resulting in the same type of motion. Thus we expect that all types of defects that give rise to pinning potentials that are sufficiently deep (e.g., voids, grain boundaries, thickness fluctuations, etc.) allow vortex core polarization switching and consequently lead to the same mobility. 
Due to the consecutive polarization switches at defects, the vortex core generally does not reach the edges of the nanowire. However, in the event it does happen, we observe that defects at the edges allow the nucleation of a vortex core of opposite polarity, as is the case in wires with edge roughness [23].

In contrast to the $0 \mathrm{~K}$ temperature we considered in the simulations, a nonzero temperature results in thermally activated depinning and finite but small velocities in a creep regime [36]. We checked the influence of temperature on the observed phenomena. However, apart from introducing nonzero velocities slightly below the depinning threshold (creep regime), no influence was seen on the domain wall mobility in the flow regime.

\section{CONCLUSION}

In conclusion, we have shown that material defects vastly influence the domain wall dynamics. The defects enable the vortex core to successively switch polarization, hindering the transverse domain wall formation. As a result the intrinsic pinning (adiabatic case) as well as the Walker breakdown (nonadiabatic case) are absent. Furthermore, at low currents the domain wall is extrinsically pinned. The successive vortex core switches give rise to a motion of the vortex core in the central region of the nanowire, as also present in perfect nanowires with a degree of nonadiabaticity equal to the Gilbert damping. This explains the consistently lower values $\beta \approx \alpha$ found in experiments based on measurements of the average velocity of vortex domain walls in nanowires.

These results show that realistic material defects have a significant influence on the domain wall mobility and not only in the creep regime. It remains to be evaluated how they influence other methods for the determination of $\beta$, but it is clear that they should be included in the evaluation of experimental data when new concepts are introduced to enhance the domain wall mobility, e.g., by spin-Hall and Rashba effects.

\section{ACKNOWLEDGMENTS}

This work is supported by the Flanders Research Foundation (B.V.d.W. and A.V.), the Academy of Finland through an Academy Research Fellowship (L.L., Project No. 268302), and through the Centres of Excellence Program (L.L., Project No. 251748), Progetto Premiale MIUR-INRIM "Nanotecnologie per la metrologia elettromagnetica" (G.D.) and MIURPRIN 2010-11 Project2010ECA8P3 “DyNanoMag”(G.D.).
[1] D. A. Allwood, G. Xiong, D. Faulkner, D. Atkinson, D. Petit, and R. P. Cowburn, Science 309, 1688 (2005).

[2] S. E. Barnes, J. Ieda, and S. Maekawa, Appl. Phys. Lett. 89, 122507 (2006).

[3] S. S. P. Parkin, M. Hayashi, and L. Thomas, Science 320, 190 (2008).

[4] S. Zhang and Z. Li, Phys. Rev. Lett. 93, 127204 (2004).

[5] Y. Tserkovnyak, H. J. Skadsem, A. Brataas, and G. E. W. Bauer, Phys. Rev. B 74, 144405 (2006).

[6] D. Berkov and J. Miltat, J. Magn. Magn. Mater. 320, 1238 (2008).

[7] A. Thiaville, Y. Nakatani, J. Miltat, and Y. Suzuki, Europhys. Lett. 69, 990 (2005).

[8] Y. Ban and G. Tatara, Phys. Rev. B 80, 184406 (2009).

[9] S. Lepadatu, A. Vanhaverbeke, D. Atkinson, R. Allenspach, and C. H. Marrows, Phys. Rev. Lett. 102, 127203 (2009).

[10] S. Lepadatu, M. C. Hickey, A. Potenza, H. Marchetto, T. R. Charlton, S. Langridge, S. S. Dhesi, and C. H. Marrows, Phys. Rev. B 79, 094402 (2009).

[11] S. Lepadatu, J. S. Claydon, C. J. Kinane, T. R. Charlton, S. Langridge, A. Potenza, S. S. Dhesi, P. S. Keatley, R. J. Hicken, B. J. Hickey, and C. H. Marrows, Phys. Rev. B 81, 020413 (2010).

[12] M. Eltschka et al., Phys. Rev. Lett. 105, 056601 (2010).

[13] L. Thomas, M. Hayashi, X. Jiang, R. Moriya, C. Rettner, and S. S. P. Parkin, Nature (London) 443, 197 (2006).

[14] L. Heyne et al., Phys. Rev. Lett. 105, 187203 (2010).

[15] S. D. Pollard, L. Huang, K. S. Buchanan, D. A. Arena, and Y. Zhu, Nat. Commun. 3, 1028 (2012).

[16] G. Meier, M. Bolte, R. Eiselt, B. Krüger, D. H. Kim, and P. Fischer, Phys. Rev. Lett. 98, 187202 (2007).
[17] M. Hayashi, L. Thomas, Y. B. Bazaliy, C. Rettner, R. Moriya, X. Jiang, and S. S. P. Parkin, Phys. Rev. Lett. 96, 197207 (2006).

[18] G. S. D. Beach, C. Knutson, C. Nistor, M. Tsoi, and J. L. Erskine, Phys. Rev. Lett. 97, 057203 (2006).

[19] L. Heyne, J. Rhensius, A. Bisig, S. Krzyk, P. Punke, M. Kläui, L. J. Heyderman, L. Le Guyader, and F. Nolting, Appl. Phys. Lett. 96, 032504 (2010).

[20] O. Boulle, G. Malinowski, and M. Kläui, Mater. Sci. Eng. 72, 159 (2011).

[21] M. Kläui, P.-O. Jubert, R. Allenspach, A. Bischof, J. A. C. Bland, G. Faini, U. Rüdiger, C. A. F. Vaz, L. Vila, and C. Vouille, Phys. Rev. Lett. 95, 026601 (2005).

[22] L. Heyne et al., Phys. Rev. Lett. 100, 066603 (2008).

[23] Y. Nakatani, A. Thiaville, and J. Miltat, Nat. Mater. 2, 521 (2003).

[24] E. Martinez, J. Phys.: Condens. Matter 24, 024206 (2012).

[25] R. L. Compton and P. A. Crowell, Phys. Rev. Lett. 97, 137202 (2006).

[26] R. L. Compton, T. Y. Chen, and P. A. Crowell, Phys. Rev. B 81, 144412 (2010).

[27] J. S. Kim et al., Phys. Rev. B 82, 104427 (2010).

[28] J. A. J. Burgess, A. E. Fraser, F. Fani Sani, D. Vick, B. D. Hauer, J. P. Davis, and M. R. Freeman, Science 339, 1051 (2013).

[29] T. Y. Chen, M. J. Erickson, P. A. Crowell, and C. Leighton, Phys. Rev. Lett. 109, 097202 (2012).

[30] A. Vansteenkiste and B. Van de Wiele, J. Magn. Magn. Mater. 323, 2585 (2011). The code can be downloaded from http://mumax.github.io/3/

[31] J. Leliaert, B. Van de Wiele, A. Vansteenkiste, L. Laurson, G. Durin, L. Dupré, and B. Van Waeyenberge, J. Appl. Phys. 115, 17D102 (2014). 
[32] B. Van de Wiele, L. Laurson, and G. Durin, Phys. Rev. B 86, 144415 (2012).

[33] H. Min, R. D. McMichael, M. J. Donahue, J. Miltat, and M. D. Stiles, Phys. Rev. Lett. 104, 217201 (2010).

[34] See Supplemental Material at http://link.aps.org/supplemental/ 10.1103/PhysRevB.89.064419 for Movies 1-6 showing the simulated movement of a domain wall in perfect and disordered nanowires.

[35] M. D. Stiles (private communication).

[36] P. J. Metaxas, J. P. Jamet, A. Mougin, M. Cormier, J. Ferré, V. Baltz, B. Rodmacq, B. Dieny, and R. L. Stamps, Phys. Rev. Lett. 99, 217208 (2007). 